\title{
Pretreatment with oral contraceptive pills to identify poor responders that may benefit from rLH supplementation during GnRH-antagonist treatment for IVF: A pilot perspective study proposal
}

\author{
SALVATORE GIZZO, ALESSANDRA ANDRISANI, MARCO NOVENTA, MICHELE GANGEMI, \\ GIOVANNI BATTISTA NARDELLI and GUIDO AMBROSINI
}

\begin{abstract}
Department of Woman and Child Health, Clinic of Gynecology and Obstetrics, University of Padua, Padua I-35128, Italy
\end{abstract}
Received September 12, 2014; Accepted July 9, 2015

DOI: $10.3892 /$ etm.2015.2734

\begin{abstract}
Controlled ovarian stimulation, using a gonadotrophin-releasing hormone $(\mathrm{GnRH})$ antagonist protocol, is a potential treatment option for women with a low response to other fertility treatments as it appears to be at least as effective as GnRH agonists (long protocol). However, previous studies have indicated that the administration of $\mathrm{GnRH}$ antagonist may cause an excessive reduction in endogenous luteinizing hormone (LH) levels. The use of recombinant $\mathrm{LH}(\mathrm{rLH})$ supplementation during ovarian stimulation is controversial. The present article proposes a future study focused on women aged $\geq 40$ years old, with the aim of identifying patients who are poor responders to GnRH-antagonist treatment that may benefit from rLH supplementation. We hypothesize that patients with suppressed hypothalamic-pituitary-axis activity may benefit from rLH supplementation, as GnRH-antagonist administration has the potential to induce a marked reduction in $\mathrm{LH}$ levels in such patients compared with that in patients that exhibit a regular recovery following the administration of oral contraceptive pills (OCPs). Furthermore, patients with hyper-responsive hypothalamic-pituitary-axis activity may be affected by 'low-gonadotropin-responsiveness', similar to that observed in
\end{abstract}

Correspondence to: Professor Salvatore Gizzo, Department of Woman and Child Health, Clinic of Gynecology and Obstetrics, University of Padua, 3 Via Nicolò Giustiniani, Padua I-35128, Italy

E-mail: ginecologia_padova@libero.it

Abbreviations: ART, assisted reproductive technique; E2, $17 \beta$ estradiol; ET, embryo transfer; GnRH, gonadotropin-releasing hormone; r-hCG, recombinant human chorionic gonadotropin; IVF, in vitro fertilization; OCP, oral contraceptive pill; r-FSH, recombinant follicular-stimulating hormone; rLH, recombinant luteinizing hormone

Key words: rLH supplementation, hypothalamic-pituitary-ovarian recovery, gonadotropin release hormone-antagonist stimulation protocol, IVF outcomes, pretreatment oral contraceptive pills patients with any mutation in the follicle-stimulating hormone (FSH) receptor, who are known to benefit from rLH supplementation. The proposed pilot study would include 120 women who are predicted to be poor responders to GnRH-antagonist treatment. All subjects will be allocated at random (using 2:1 computerized randomization) into two study groups: Group A (OCP-treated) and group B (control). For all patients, the serum values of FSH, LH and $17 \beta$ estradiol (E2) will be detected on day 3 of the menstrual cycle preceding OCP treatment (baseline) and at day 4 following OCP treatment. The $\Delta$-variation from baseline levels for all markers, the FSH/LH ratio and the E2/FSH ratio will be determined. $\Delta$-variation from the baseline of the FSH and LH values will be used to further categorize group A patients into subgroups A1-4, based on respective quartile numbers (Q1-4). Patients admitted to each of the four subgroups A1-4, based on their FSH quartile, will be selected at random to receive rLH supplementation (ratio, 1:1) during ovarian stimulation. If the resulting data are able to identify women that may benefit from rLH supplementation during ovarian stimulation, a large part of inconclusive evidence regarding rLH supplementation will be clarified. If patients supplemented with rLH (according to abnormal recovery of hypothalamic-pituitary-axis activity after OCP treatment) exhibit an improved ovarian response during in vitro fertilization (IVF) and subsequent pregnancy rate, the pre-IVF OCP test could be adopted as a useful tool for improving the success rate of assisted reproductive technologies in poorly-responding patients.

\section{Introduction}

Controlled ovarian stimulation using a gonadotrophin-releasing hormone $(\mathrm{GnRH})$ antagonist is a potential treatment option for patients with a low response to fertility treatments, as it appears to be at least as effective as $\mathrm{GnRH}$-agonist treatment (long protocol) (1).

Controlled ovarian stimulation with a GnRH antagonist offers a number of advantages compared with the long agonist protocol, including a reduction in the overall duration of the treatment, the absence of peri-menopausal symptoms as a result of pituitary desensitization, no risk of inadvertent $\mathrm{GnRH}$ 
agonist administration at the early stages of pregnancy, no risk of ovarian cyst formation during the mild luteal phase and reduced doses of gonadotropins (2).

Therefore, in low responders, ovarian stimulation without pituitary suppression may induce asynchronous follicular development with a limited degree of potential follicle development. Furthermore, in patients that are predicted to be poor responders, for example with elevated serum levels of basal follicle-stimulating hormone ( $\mathrm{FSH}$ ) due to a decreased ovarian reserve, antral follicle sizes during the early follicular phase are often markedly heterogeneous. This phenomenon is associated with the early exposure of FSH-sensitive follicles to gradient FSH concentrations during the previous luteal phase.

A potential disadvantage of GnRH-antagonist protocols is that stimulation is generally initiated on days 2 or 3 of the menstrual cycle, which increases the difficulty of planning stimulation and laboratory activities. However, cycle scheduling may be used to avoid having to retrieve oocytes at the weekend and to distribute the workload equally throughout the week, thereby reducing the disturbance of incubators and the associated negative impact on embryonic development. Furthermore, it can reduce the amount of unplanned laboratory work, which may adversely affect the concentration and efficiency of laboratory staff (3).

Oral contraceptive pills (OCPs) and synthetic progestogens have previously been used to schedule the ovarian stimulation cycle (4-6). Estrogens primarily inhibit FSH secretion, whereas progestogens primarily control luteinizing hormone (LH) secretion. The mechanisms underlying this process have not been well defined; however, it has been postulated that the gestagen component of OCPs may exert a negative impact on endometrial receptivity in the subsequent cycle. Alternatively, low concentrations of LH following OCP pretreatment may impair oocyte quality or endometrial receptivity if ovarian stimulation is performed using recombinant $\mathrm{FSH}(\mathrm{rFSH})$ but no LH is administered (4-6).

According to in vitro fertilization (IVF) stimulation protocols, GnRH antagonists are administered to prevent a premature pituitary LH surge, which exerts a detrimental effect in patients that undergo IVF. Treatment with GnRH antagonists may result in reduced gonadotropin levels. In order to facilitate follicle growth, FSH is administered exogenously, whereas LH is not typically administered. Although GnRH antagonists prevent surges in LH levels, there is evidence that the administration of GnRH analogs may result in an excessive reduction in endogenous LH levels, particularly in older women $(7,8)$. A number of studies have been performed to investigate whether the additional administration of recombinant $\mathrm{LH}(\mathrm{rLH})$ with $\mathrm{rFSH}$ is able to improve the ovarian stimulation cycle outcome $(9,10)$. Few studies have analyzed this factor in the context of GnRH antagonist treatment, and the results of these trials obtained in older women ( $>35$-years-old) are inconsistent (11).

The use of rLH supplementation during ovarian stimulation, as a part of an assisted reproductive technique (ART), has not been demonstrated to increase pregnancy success rate, and previous studies have reported conflicting results in women aged $\geq 35$ years. Previous results do not support the hypothesis that the addition of rLH increases the rate of pregnancy in unselected patients treated with an ART protocol consisting of $\mathrm{rFSH}$ with a $\mathrm{GnRH}$ antagonist. There may be a potential benefit associated with the use of rLH supplementation during ovarian stimulation in women who have a poor response or are of an advanced age (12).

Therefore, it may be valuable to conduct a study focused on women aged $\geq 40$ years, with the aim of determining if women may be able to benefit from rLH supplementation during ovarian stimulation and identifying the cohort of women that would benefit, in order to improve IVF outcome.

In previous literature, the only study conducted in women $>35$ years old that received a $\mathrm{GnRH}$ antagonist protocol, postulated that the degree of hypothalamic-pituitary-ovarian recovery following pretreatment with OCP administration, may be used to identify patients that could benefit from rLH supplementation (13). However, to date no such perspective randomized study has been conducted to investigate this topic.

\section{Materials and methods}

Presentation of the hypothesis. The currently proposed hypothesis is based on rationale that, in older women that are predicted to respond poorly to $\mathrm{GnRH}$ antagonist protocols, the extent to which serum levels of endogenous gonadotrophins are returned to normal by action of the hypothalamic-pituitary-ovarian axis following pretreatment with OCPs may be useful for identifying whether women may benefit from rLH supplementation, and if so, which ones.

In certain cases, older women who are predicted to be poor responders exhibit an altered hypothalamic-pituitary-ovarian regulatory mechanism due to the dysregulation of E2 feedback. The aberrant responsiveness of the hypothalamic-pituitary axis to estrogen feedback and the subsequent generation of abnormal patterns of gonadotropin release (normal, attenuated or increased) may accelerate spontaneous ovarian follicular depletion and reduce the likelihood of a good ovarian responsiveness during controlled ovarian hyperstimulation.

We hypothesize that patients with suppressed activity of the hypothalamic-pituitary axis may benefit from rLH supplementation, as GnRH antagonist administration during ovarian stimulation has the potential to induce a marked reduction in LH levels in such patients compared with that in patients with a regular recovery of the activity of this axis following OCP.

Furthermore, we hypothesize that patients with hyper-responsiveness of hypothalamic-pituitary-axis activity following OCP may be affected by 'low gonadotropin responsiveness' comparable to that in patients with a mutation in the FSH receptor (14). Thus, such cases may benefit from the ability of rLH supplementation to potentiate exogenous FSH activity in theca and granulosa cells.

Therefore, the primary aim of the currently proposed study is to determine whether 1 month pretreatment with OCP is useful for detecting the cohort of women in a pool of estimated poor responders aged $>40$ years that may benefit from rLH supplementation during the ovarian stimulation cycle according to hypothalamic-pituitary-ovarian recovery after OCP administration. A secondary aim of the proposed study is to detect the most effective serum markers for assessing the cohort of patients that may benefit from rLH administration during ovarian stimulation. 


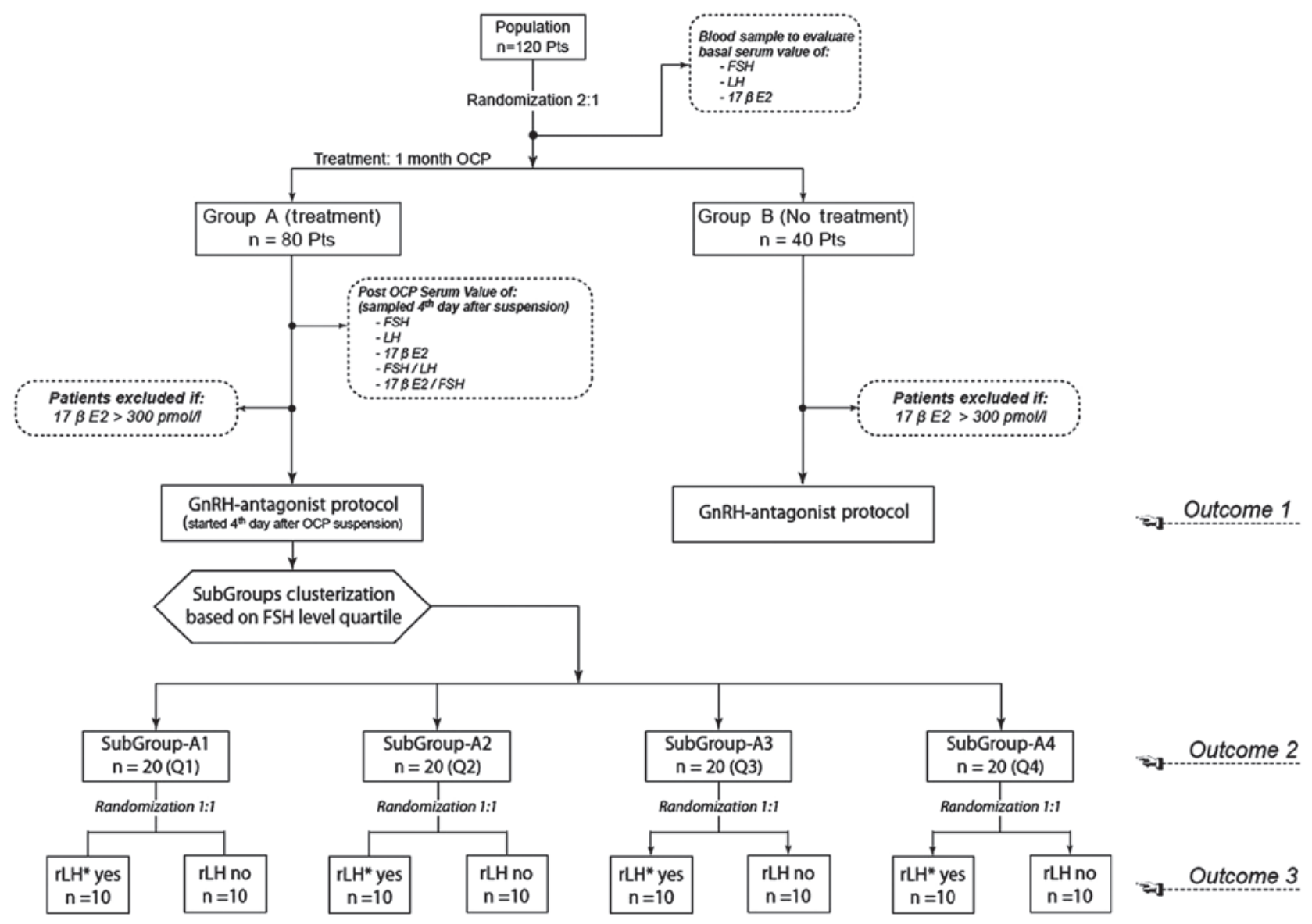

Figure 1. Flow chart of the study protocol. FSH, follicle-stimulating hormone; LH, luteinizing hormone; rLH , recombinant luteinizing hormone; E2, estradiol; OCP, oral contraceptive pill; GnRH, gonadotrophin-releasing hormone.

Testing the hypothesis. The proposed pilot study will include 120 women aged between 40 and 50 years, that are predicted to be poor responders according to the Bologna Criteria (15), with an ovarian biological age higher than their chronological age (16-18). Patients who have not previously undergone a first fresh non-donor IVF cycle for primary infertility without rLH supplementation during ovarian stimulation will be excluded.

All enrolled patients will be properly informed regarding the aims of the study, and will be required to agree to these aims and to the use of their data according to the Italian Law for Privacy 675/96 prior to enrolment.

Patients with any of the following characteristics will be excluded: History of smoking, deep endometriosis with elevated CA125 serum value (19), previous ART cycle in the preceding 3 months, BMI $>30$, karyotype abnormalities, mutations of the cystic fibrosis gene, acquired or inherited thrombophilia and immunological disorders, previous chemotherapy and/or radiotherapy for neoplasia treatment and marked qualitative and quantitative alteration in the semen used for fertilization, according to World Health Organization guidelines (20).

In cases of benign uterine lesions, such as endometrial polyps, submucous myomas, intrauterine synechiae and/or uterine septus, patients will be considered eligible for the study if they have undergone hysteroscopic-adequate treatment at least 3 months previously (21-23).

All women, accurately selected according to the exclusion criteria, will be assigned at random (using 2:1 computerized randomization) to one of two study groups: Group A (OCP-treated) or group B (control).

Group A patients will be pre-treated with OCPs containing $2 \mathrm{mg}$ dienogest and $0.03 \mathrm{mg}$ ethinyl estradiol (Effiprev ${ }^{\circledR}$; Effik Italia SpA, Milan, Italy) for 21 days during the menstrual cycle prior to the scheduled IVF/intra-cytoplasmic sperm injection.

For all patients, serum values of FSH, LH and E2 will be determined on day 3 of the cycle preceding OCP, considered as the basal values, and at the beginning of stimulation (4 days after the final OCP for group A patients and 4 days after the initiation of menses for group B patients).

Single absolute numbers and $\Delta$-variation from baseline (expressed as a percentage) will be considered for all markers, the $\mathrm{FSH} / \mathrm{LH}$ ratio and the E2/FSH ratio in the blood samples.

$\Delta$-variation from the baseline FSH and LH values will be used to categorize patients into one of four quartiles (Q1, Q2, Q3 and Q4), in a similar manner to the study proposed by Schmitz et al (24).

Patients will be classified based on the following criteria: $\mathrm{Q} 1$, patients with negative baseline variation $>25 \%$; 2 , patients with negative baseline variation between 5 and $25 \%$; Q3, patients with positive baseline variation between 5 and $25 \%$; and Q4, patients with positive baseline variation $>25 \%$.

According to their obtained FSH quartile, patients will be admitted to the subgroup A1 in cases of Q1, to subgroup A2 in cases of Q2, to subgroup A3 in cases of Q3 and to subgroup A4 in cases of Q4. 
Patients admitted to each of the subgroups A1-4 (n=20 per subgroup) will be secondly randomized to determine whether they will receive rLH supplementation (ratio, 1:1) during ovarian stimulation, starting from day 4 of stimulation. A detailed flow chart of the proposed study protocol is shown in Fig. 1.

Patients in groups A and B will be treated with GnRH-antagonist (short protocol) stimulation according to our Units Protocol $(25,26)$. All stimulation cycles will be performed using 350 IU rFSH (Gonal F; Merck Serono S.A., Geneva, Switzerland) daily for the first 5 days. Subsequent adjustments to the treatment will be decided by the clinicians according to ovarian response and various biochemical and ultrasound features. In addition, $0.25 \mathrm{mg} \mathrm{GnRH}$ antagonist (Ganirelix; MSD Italia S.R.L., Rome, Italy) will be administered daily, starting from the ultrasonographic detection of at least one follicle of $>14 \mathrm{~mm}$ diameter and continued until human chorionic gonadotropin (hCG) administration.

Starting at day 6 of stimulation, all patients will undergo serum sampling for the measurement of hormone levels (17 $\beta$ estradiol, progesterone and LH) and pelvic ultrasound.

After an adequate number of follicles ( $\geq 3$ follicles with a diameter of $>18 \mathrm{~mm}$ ) have been detected, $250 \mu \mathrm{g}$ recombinant hCG (r-hCG) (Ovitrelle; Merck Serono S.A.) will be administered to induce ovulation.

Oocyte retrieval will be conducted at $36 \mathrm{~h}$ after hCG administration. Oocytes will be fertilized according to standard IVF techniques.

Between 1 and 3 embryos will be transferred on days 2 to 3 following oocyte retrieval. The number of embryos transferred will be determined on the basis of the age of the patient and embryo quality.

Furthermore, $400 \mathrm{mg}$ vaginal progesterone (Progeffik; Effik Italia SpA) will be administered daily as a luteal support until day 14 after oocyte retrieval, and the treatment will be discontinued in the event of a negative $\beta$-hCG serum test.

Pregnancy will be confirmed by the detection of increased $\beta$-hCG concentrations at week 2 after embryo transfer (ET) and with sonographic observation of an intrauterine gestational sac at weeks 3-4 after ET. Continuing pregnancy will be confirmed by the detection of an embryo heart beat with transvaginal sonography.

The primary aim of the proposed study is to compare the following parameters between groups A and B: Number of IVF cycles that are cancelled prior to ovarian stimulation (due to a basal E2 value of $>300 \mathrm{pmol} / \mathrm{l}$ ) and during ovarian stimulation (due to $<3$ follicles with a diameter of $>14 \mathrm{~mm}$ ); total dose of rFSH administered during ovarian stimulation (IUs); length of stimulation (days); total number of follicle and number of follicles $>14 \mathrm{~mm}$ in diameter; total number of retrieved oocytes and number of mature oocytes; number and quality of obtained embryos, endometrial thickness at oocyte retrieval; pregnancy rate; and continuing pregnancy rate.

A secondary aim of the study is to compare the following parameters among the four A subgroups: Total dose of rFSH administered during ovarian stimulation (IUs), length of stimulation (days), total number of follicle and number of follicles $>14 \mathrm{~mm}$ in diameter, total number of retrieved oocytes and number of mature oocytes, number and quality of obtained embryos, endometrial thickness at oocyte retrieval, pregnancy rate and continuing pregnancy rate in order to detect which, if any, FSH quartile will be a predictor of an improved IVF outcome.

Finally, a third intended outcome is to detect whether rLH supplementation improves the IVF outcomes in each subgroup-A patient, determine in which of the interquartile subgroups A1-4 the rLH supplementation produces the most improved results and identify whether variations in other serum markers after OCP have a greater predictive capability than the FSH serum value in the detection of patients that may benefit from rLH supplementation. Detailed steps of the proposed study protocol are summarized in Fig. 1.

Statistical analysis will be performed using SPSS software for Windows, version 19.0 (IBM SPSS, Armonk, USA), applying parametric and non-parametric tests when appropriate. The Kolmogorov-Smirnov test will be used to assess the normality of distribution. Continuous variables will be expressed as absolute numbers, the mean \pm standard deviation, and will be analyzed using Student's t-test or analysis of variance when appropriate. Categorical variables will be expressed as percentages and analyzed using the $\chi^{2}$ test or Fisher's exact test. $\mathrm{P}<0.05$ will be considered to indicate a statistically significant difference.

\section{Discussion}

If the current hypothesis is confirmed, it should facilitate the identification of women that could benefit from rLH supplementation during ovarian stimulation, among the cohort of predicted poor responders. Consequently, previous inconclusive results concerning rLH supplementation may be clarified.

If patients supplemented with rLH (according to abnormal recovery of the activity of the hypothalamic-pituitary axis following OCP treatment) display improvements in terms of ovarian response during IVF and subsequently pregnancy rate, the OCP test pre-IVF may be considered to be a useful tool for improving the success of ARTs in poor responders, reducing cost and patient stress.

It is reasonable to expect that the number of patients that consent to the 1-month OCP study will be high, as the proposed study involves a low-expense, well-tolerated intervention, with no obvious contraindications.

If the predictive efficacy of the OCP-test is confirmed, its use in patients prior to GnRH antagonist protocols to detect patients requiring $\mathrm{rLH}$ supplementation during ovarian stimulation may be extended to a large scale population.

\section{References}

1. Kim CH, Jeon GH, Cheon YP, Jeon I, Kim SH, Chae HD and Kang BM: Comparison of GnRH antagonist protocol with or without oral contraceptive pill pretreatment and GnRH agonist low-dose long protocol in low responders undergoing IVF/intracytoplasmic sperm injection. Fertil Steril 92: 1758-1760, 2009.

2. Devroey P, Aboulghar M, Garcia-Velasco J, Griesinger G, Humaidan P, Kolibianakis E, Ledger W, Tomás C and Fauser BC: Improving the patient's experience of IVF/ICSI: A proposal for an ovarian stimulation protocol with GnRH antagonist co-treatment. Hum Reprod 24: 764-774, 2009.

3. Al-Inany HG, Youssef MA, Aboulghar M, Broekmans F, Sterrenburg M, Smit J and Abou-Setta AM: Gonadotrophinreleasing hormone antagonists for assisted reproductive technology. Cochrane Database Syst Rev 5: CD001750, 2011. 
4. Griesinger G, Kolibianakis EM, Venetis C, Diedrich K and Tarlatzis B: Oral contraceptive pretreatment significantly reduces ongoing pregnancy likelihood in gonadotropin-releasing hormone antagonist cycles: An updated meta-analysis. Fertil Steril 94: 2382-2384, 2010.

5. Kolibianakis EM, Papanikolau EG, Camus M, Tournaye H, Van Steirteghem AC and Devroey P: Effect of oral contraceptive pill pretreatment on ongoing pregnancy rates in patients stimulated with $\mathrm{GnRH}$ antagonists and recombinant FSH for IVF. A randomized controlled trial. Hum Reprod 21: 352-357, 2006.

6. Rombauts L, Healy D and Norman RJ: Comparative randomized trial to assess the impact of oral contraceptive pretreatment on follicular growth and hormone profiles in $\mathrm{GnRH}$ antagonist-treated patients. Hum Reprod 21: 95-103, 2006.

7. Gizzo S, Andrisani A, Noventa M, Manfè S, Oliva A, Gangemi M, Nardelli GB and Ambrosini G. Recombinant LH supplementation during IVF cycles with a GnRH-antagonist in estimated poor responders: A cross-matched pilot investigation of the optimal daily dose and timing. Mol Med Rep 12: 4219-4229, 2015.

8. Alviggi C, Clarizia R, Mollo A, Ranieri A and De Placido G: Who needs LH in ovarian stimulation? Reprod Biomed Online: doi: 10.1016/S1472-6483(11)60007-2.

9. Gizzo S, Quaranta M, Andrisani A, Bordin L, Vitagliano A, Esposito F, Venturella R, Zicchina C, Gangemi M and Noventa M Serum stem cell factor assay in elderly poor responder patients undergoing IVF: A new biomarker to customize follicle aspiration cycle by cycle. Reprod Sci: July 7, 2015 (Epub ahead of print).

10. Hill MJ, Levens ED, Levy G, Ryan ME, Csokmay JM, DeCherney AH and Whitcomb BW. The use of recombinant luteinizing hormone in patients undergoing assisted reproductive techniques with advanced reproductive age: a systematic review and meta-analysis. Fertil Steril: February 24, 2012 (Epub ahead of print) doi: 10.1016/j.fertnstert.2012.01.130.

11. König TE, van der Houwen LE, Overbeek A, Hendriks ML, Beutler-Beemsterboer SN, Kuchenbecker WK, Renckens CN, Bernardus RE, Schats R, Homburg R, et al: Recombinant LH supplementation to a standard GnRH antagonist protocol in women of 35 years or older undergoing IVF/ICSI: A randomized controlled multicentre study. Hum Reprod 28: 2804-2812, 2013.

12. König TE, van der Houwen LE and Lambalk CB: Recombinant LH supplementation in women of 35 years and older undergoing IVF? Fertil Steril 98: e10-e11, 2012.

13. Schmitz C, Bocca S, Beydoun H, Stadtmauer L and Oehninger S: Does the degree of hypothalamic-pituitary-ovarian recovery after oral contraceptive pills affect outcomes of IVF/ICSI cycles receiving $\mathrm{GnRH}$-antagonist adjuvant therapy in women over 35 years of age? J Assist Reprod Genet 29: 877-882, 2012.

14. Desai SS, Roy BS and Mahale SD: Mutations and polymorphisms in FSH receptor: functional implications in human reproduction. Reproduction 146: R235-248, 2013.

15. Ferraretti AP, La Marca A, Fauser BC, Tarlatzis B, Nargund G and Gianaroli L; ESHRE Working Group On Poor Ovarian Response Definition: ESHRE consensus on the definition of 'poor response' to ovarian stimulation for in vitro fertilization: The Bologna criteria. Hum Reprod 26: 1616-1624, 2011.
16. Patrelli TS, Gizzo S, Sianesi N, Levati L, Pezzuto A, Ferrari B and Bacchi Modena A: Anti-Müllerian hormone serum values and ovarian reserve: Can it predict a decrease in fertility after ovarian stimulation by ART cycles? PLoS One 7: e44571, 2012.

17. Gizzo S, Andrisani A, Esposito F, Oliva A, Zicchina C, Capuzzo D, Gangemi M and Nardelli GB: Ovarian reserve test: An impartial means to resolve the mismatch between chronological and biological age in the assessment of female reproductive chances. Reprod Sci 21: 632-639, 2014.

18. Litta P, D'Agostino G, Conte L, Saccardi C, Cela V, Angioni S and Plebani M: Anti-Müllerian hormone trend after laparoscopic surgery in women with ovarian endometrioma. Gynecol Endocrinol 29: 452-454, 2013.

19. Patrelli TS, Berretta R, Gizzo S, Pezzuto A, Franchi L, Lukanovic A, Nardelli GB and Modena AB: CA 125 serum values in surgically treated endometriosis patients and its relationships with anatomic sites of endometriosis and pregnancy rate. Fertil Steril 95: 393-396, 2011.

20. World Health Organization: WHO Laboratory Manual for the Examination of Human Semen and Sperm-Cervical Mucus Interaction. Cambridge University Press, Cambridge, UK, 1999.

21. Litta P, Cosmi E, Saccardi C, Esposito C, Rui R and Ambrosini G: Outpatient operative polypectomy using a $5 \mathrm{~mm}$-hysteroscope without anaesthesia and/or analgesia: Advantages and limits. Eur J Obstet Gynecol Reprod Biol 139: 210-214, 2008.

22. Saccardi C, Conte L, Fabris A, De Marchi F, Borghero A, Gizzo S and Litta P: Hysteroscopic enucleation in toto of submucous type 2 myomas: Long term follow up in women affected by menorrhagia. J Minim Invasive Gynecol 21: 426-430, 2014

23. Litta P, Spiller E, Saccardi C, Ambrosini G, Caserta D and Cosmi E: Resectoscope or Versapoint for hysteroscopic metroplasty. Int J Gynaecol Obstet 101: 39-42, 2008.

24. Schmitz C, Bocca S, Beydoun H, Stadtmauer L and Oehninger S: Does the degree of hypothalamic-pituitary-ovarian recovery after oral contraceptive pills affect outcomes of IVF/ICSI cycles receiving GnRH-antagonist adjuvant therapy in women over 35 years of age? J Assist Reprod Genet 29: 877-882, 2012.

25. Gizzo S, Andrisani A, Noventa M, Quaranta M, Esposito F, Armanini D, Gangemi M, Nardelli GB, Litta P, D'Antona D and Ambrosini G. Menstrual cycle length: a surrogate measure of reproductive health capable of improving the accuracy of biochemical/sonographical ovarian reserve test in estimating the reproductive chances of women referred to ART. Reprod Biol Endocrinol: April 10, 2015 (Epub ahead of print) doi: 10.1186/s12958-015-0024-1.

26. Gizzo S, Andrisani A, Esposito F, Noventa M, Di Gangi S, Angioni S, Litta P, Gangemi M and Nardelli GB. Which luteal phase support is better for each IVF stimulation protocol to achieve the highest pregnancy rate? A superiority randomized clinical trial. Gynecol Endocrinol: September 30, 2014 (Epub ahead of print). 